\title{
Novel Sources for Synchrotron Light
}

\author{
E. WECKERT \\ HASYLAB at DESY, Notkestrasse 85, 22603 Hamburg, Germany
}

\begin{abstract}
During the past decades synchrotron radiation has become an amazingly versatile tool for a very large range of applications ranging from basic physics, materials science, condensed matter physics, chemistry to life sciences, just to mention a few. The properties most widely exploited are high brilliance or flux, tunability, polarisation, and temporal resolution. More recently with the advent of third generation synchrotron sources providing very small source sizes partially coherent radiation even in the $\mathrm{X}$-ray regime is available which enables a large number of new experiments. However, as far as it concerns pulse length and coherence traditional storage ring based sources encounter limits that cannot easily be surpassed. Having also these applications in mind new concepts have been developed. Mainly in the US plans are worked out for linac driven storage rings. In these devices electrons circulate only once in a storage ring after they have been accelerated by a high current/power linac. The emittance of such a source would be considerably smaller than that of a normal storage ring of the same size. A totally coherent beam down in wavelengths to the $\AA$-regime will be delivered by so-called X-ray free electron lasers proposed in the US (Stanford), in Germany (DESY, Hamburg), and in Japan (Spring8/KEK). In these systems the beam of a linac is directly fed through very long undulators where X-rays are generated according to the so-called self-amplified spontaneous emission process. The radiation after such a device will be fully coherent in the transverse direction and the pulse length will be in the $0.1 \mathrm{ps}$ regime. These are properties unseen so far. The peak (average) brilliance of such an X-ray free electron laser will be about ten (five) orders of magnitude higher compared to the most advanced present day synchrotron radiation sources.
\end{abstract}

PACS numbers: 52.59.-f

\section{Introduction}

During the last one or two decades a number of new synchrotron radiation sources has been constructed. Most of them are considered to be sources of the so-called 3rd generation like for example the European Synchrotron Radiation Facility (ESRF) in Grenoble, the Advanced Photon Source (APS) at Argonne (USA), 
Spring8 in Japan, ELETTRA close to Trieste (Italy), BESSYII in Berlin, and the sources at Maxlab in Lund (Sweden). ${ }^{*}$ Synchrotron radiation sources of the 3rd generation are characterised by a very small horizontal emittance $\epsilon_{x}=\sigma_{x} \sigma_{x^{\prime}}$ with $\sigma_{x}$ and $\sigma_{x^{\prime}}$ the horizontal size and divergence of the electron beam, respectively. This low emittance is the prerequisite for the efficient use of undulators which provide a significantly higher brilliance $B=F /\left(4 \pi^{2} \sigma_{\mathrm{T} x} \sigma_{\mathrm{T} x^{\prime}} \sigma_{\mathrm{T} y} \sigma_{\mathrm{T} y^{\prime}}\right)$ compared to the wiggler based sources characteristic of the radiation sources of the 2 nd generation. Hereby, $F$ denotes the total flux normally given in $\mathrm{ph} /(\mathrm{s} 0.1 \% \mathrm{BW})$, $x$ and $y$ denote the horizontal and vertical direction, respectively, the index "T" indicates "total", since it is the total source size or divergence being a convolution of the electron beam properties with the radiation properties of a single electron that matters for the brilliance. The brilliance $B$ is measured in $\mathrm{ph} /\left(\mathrm{s} \mathrm{mrad}^{2} \mathrm{~mm}^{2}\right.$ $0.1 \% \mathrm{BW}$ ) that means as the number of photons in a certain solid angle normalised to the total source size and for a given energy band width (BW). Undulator radiation is inherently already very well collimated, therefore most of the experiments use only the central part of the whole radiation cone. For a given brilliance the heat load on the optical components is significantly smaller than for wiggler insertion devices. Undulators are best suited for the investigation of small and if focused also for extremely small samples. For the investigation of large samples wiggler radiation sources are the best choice in most cases.

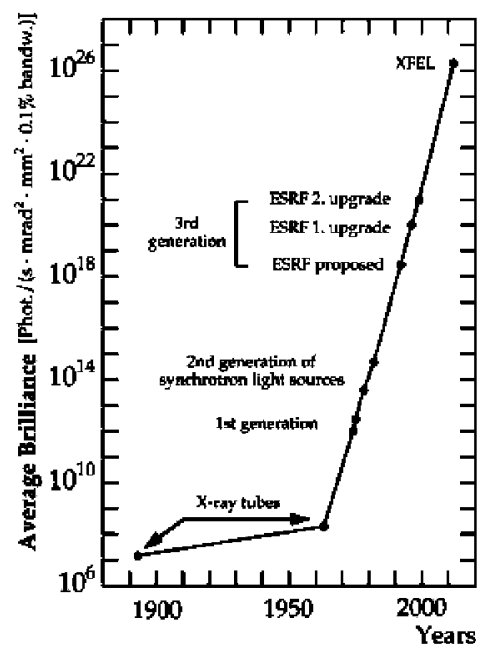

Fig. 1. Development of the average brilliance versus time since the discovery of X-rays [1]. The 2 nd generation sources using wigglers are in the $10^{15}$ brilliance range, the undulators of 3rd generation sources are between $10^{19}$ and $10^{21}$. Significant higher brilliances can only be achieved with linear accelerator driven sources.

* Of course this list is far from being complete. 
In Fig. 1 the development of the average brilliance versus time since the discovery of X-rays is shown. From about 1960 on there was an increase in about three orders of magnitude every decade.

The emittance is a quantity typical of each storage ring that adjusts itself to an equilibrium value after a so-called damping time, which is in the order of milliseconds. It depends according to $\epsilon_{x} \propto E^{2} / N_{\mathrm{DP}}^{3}$ (e.g. [2]) on the electron energy $E$, the number of dispersive elements (dipole magnets) $N_{\mathrm{DP}}$ and some lattice specific functions. In order to provide a small emittance high electron energy storage rings have to have a large number of dipole magnets. This is the reason why modern high energy storage rings are of about $1 \mathrm{~km}$ circumference.

In order to further lower the emittance storage rings have to increase significantly in size until the diffraction limit is reached. This is the case if the electron beam is that small and parallel that the source properties are dominated by the emittance behaviour of a single electron. A further increase in brilliance can only be achieved by linear accelerator driven free electron lasers (FELs).

In the following several new 3rd generation synchrotron radiation sources will be presented very briefly that are just commissioned, under construction or planned. In a second part the possibility of linac driven radiation sources will be presented with special emphasise on the DESY FEL-plans. In the final section the DESY plans for upgrades of the present storage ring facilities will be discussed.

\section{New 3rd generation storage rings}

It is obviously not possible to deal with all storage rings here that are planned and under construction at the moment. For this reason we will concentrate on three projects in the lower energy regime. These are the Swiss-Light-Source (SLS) which went just into operation, the Soleil project in France and the Diamond project in England. The last two projects are at the begin of their construction. The machine group at the ESRF has exploited to possibilities of an Ultimate Storage Ring (USR). This plan will also be briefly presented.

The very successful running present 3rd generation storage rings will not be discussed since they are already very familiar to many scientists using them regularly.

\subsection{New 3rd generation sources at lower energies}

The SLS, which is situated at the Paul-Scherrer-Institute in Villingen, has been commissioned very successfully during the last year and serves beam to a number of experimental stations meanwhile. Most actual information is available under the SLS-home page. Some characteristic storage ring parameters are compared in Table I. At SLS it is anticipated to obtain a quite high brilliance in the $\mathrm{X}$-ray regime at energies around $12 \mathrm{keV}$ by the use of undulators of very small magnetic gaps. The inherent electron beam life time reduction of small gap undulators 
is solved by a continuous injection mode (toping up mode) that keeps the electron current almost constant. The SLS storage ring with its extremely long straight sections is ideally suited for the production of undulator radiation in the softer part of the electromagnetic spectrum. A high flux at high X-ray photon energies, e.g. for materials science applications, will be provided by multipole wigglers or wavelength shifters.

TABLE I

Comparison of some key parameters of new synchrotron radiation sources. The critical energy corresponds to the radiation spectrum emitted from the bending magnets. The brilliances are given in $\mathrm{ph} /\left(\mathrm{s} \mathrm{mm}^{2} \mathrm{mrad}^{2} 0.1 \% \mathrm{BW}\right)$. The brilliance values for Soleil correspond to $2.75 \mathrm{GeV}$ electron energy and for the $20 \mathrm{keV}$ value for a $4 \mathrm{~mm}$ gap undulator. The information given above has been collected from the corresponding WEB-pages (SLS: www1.psi.ch/www_sls_hn; Soleil: www.soleil.u-psud.fr; Diamond: www.diamond.ac.uk).

\begin{tabular}{l|c|c|c}
\hline \hline & SLS & Soleil & Diamond \\
\hline energy [GeV] & 2.4 & 2.5 & 3 \\
\hline circumference [m] & 288 & 337 & 560 \\
\hline current [mA] & 400 & 500 & 300 \\
\hline emittance [nm rad] & 4.4 & 3 & 2 \\
\hline critical energy [keV] & 5.4 & 6.5 & 8.4 \\
\hline max. brilliance & $10^{20}$ & $>10^{20}$ & $2 \times 10^{20}$ \\
\hline brilliance@10 keV & $2 \times 10^{18}$ & $10^{19}$ & $5 \times 10^{19}$ \\
\hline brilliance@20 keV & $1 \times 10^{17}$ & $10^{19}$ & $4 \times 10^{18}$ \\
\hline straight sections & $2 \times 15 \mathrm{~m}$ & $3 \times 14 \mathrm{~m}$ & $4 \times 8 \mathrm{~m}$ \\
for insertion & $2 \times 8 \mathrm{~m}$ & $11 \times 7 \mathrm{~m}$ & $18 \times 5 \mathrm{~m}$ \\
devices & $2 \times 6 \mathrm{~m}$ & &
\end{tabular}

The French Soleil synchrotron project to be realised at the Saclay site south of Paris is similar to the SLS. The energy as well as the circumference are slightly higher providing about twice the number of straight sections compared to the SLS. Also Soleil will have space for very long and powerful undulators in the VUV regime. In order to serve also the $\mathrm{X}$-ray community with high brilliance radiation undulators down to $4 \mathrm{~mm}$ magnetic gap are envisaged like at SLS. Again for high flux at high photon energies ( $>20 \mathrm{keV}$ ) multipole wigglers will be used. First user experiments at Soleil are expected to be carried out in 2006.

The British Diamond synchrotron radiation facility will be built at Didcod close to the Rutherford Appleton Laboratory near Oxford. This will be already a quite large facility with more than 20 insertion device positions and a large number of possible bending magnet stations. Due to its electron energy of $3 \mathrm{GeV}$ and the very small emittance undulators at X-ray energies suitable for protein crystallography provide brilliances close to standard devices $(1.65 \mathrm{~m})$ insertion at 
the ESRF. Due to the higher electron energy compared to SLS and Soleil these brilliance values can be obtained at quite decent undulator gap values of about $10 \mathrm{~mm}$. The high energy photon spectrum can again be only accessed by high field multipole wigglers. The first beamlines of Diamond are scheduled to become operational in 2007.

\subsection{The ultimate storage ring}

The machine group at the ESRF has carried out a study to explore the limits of storage ring based light sources [3]. The aim was to find technical achievable parameters for a storage ring that will be close to the diffraction limit. In the study the circumference was limited to $2 \mathrm{~km}$ for budget reasons. An energy of $7 \mathrm{GeV}$ was assumed in order to provide optimum (tunable) undulator performance up to energies of $50 \mathrm{keV}$ on harmonics no. 1-5 with a minimum gap of $11 \mathrm{~mm}$. For undulators at higher photon energies in-vacuum devices with smaller gaps would be required if extremely high brilliances are needed. The maximum current was limited to $500 \mathrm{~mA}$. The emittance of such a storage ring would be $0.3 \mathrm{~nm}$ rad. About 40 positions for $7 \mathrm{~m}$ long insertion devices would be available. The brilliance of the undulators in such a storage ring would be in the $10^{22}$ range even up to photon energies of $30 \mathrm{keV}$ (see Fig. 2). A further reduction of the emittance by the systematic use of damping wigglers was considered not to be worthwhile.

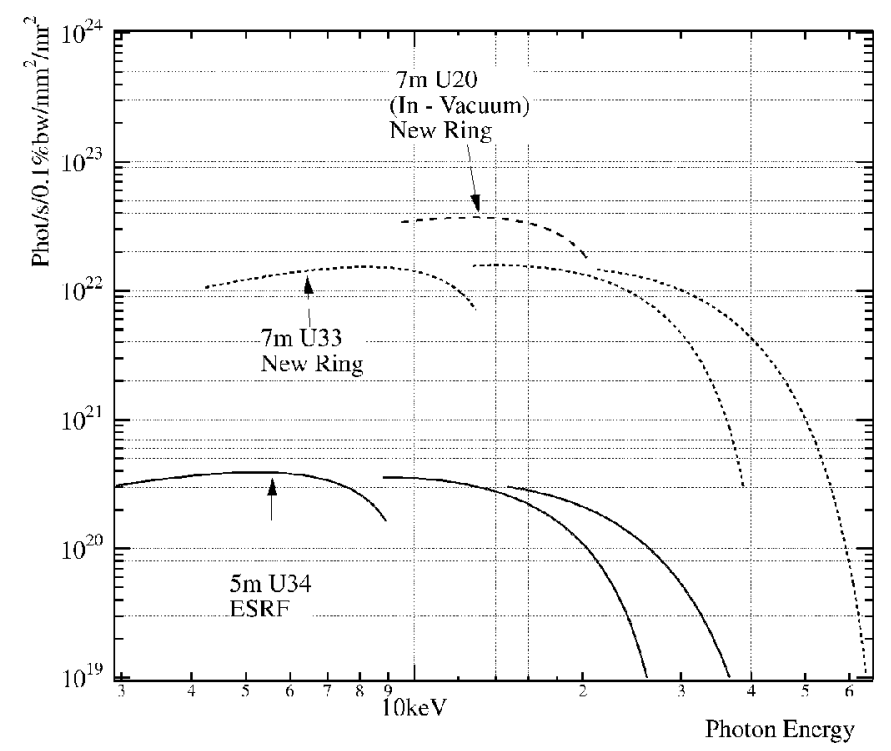

Fig. 2. Brilliance of undulators at the ultimate storage ring compared to the best values of ESRF at present [3] (the figure is reproduced from the ESRF Highlights 2000, with ESRF permission). 


\section{Linear accelerator driven radiation sources}

The emittance of a storage ring is an equilibrium value, as already mentioned above, that appears after several thousand turns of the electron bunches after injection. The same holds for the electron bunch length and therefore the shortest possible radiation pulses. However, during a single turn the electron beam quality is almost not degraded. Based on this fact a new synchrotron radiation scheme, namely the energy recovery linac driven storage ring, has been proposed. Another possibility is to feed the electron beam from a linac directly into a long undulator as it is done for free electron lasers.

\subsection{Energy recovery linac driven storage rings}

In an energy recovery linac (ERL) a high current electron beam is accelerated in a linac. The emittance at the end of the linac depends only on the quality of the electron gun to generate the bunches and on the linac itself. In general these $\epsilon_{x}$-values are considerable smaller than the best values that can be obtained by storage rings. The low emittance electron beam is then guided through a storage ring with a number of undulators. After one turn in the storage ring the electron bunches are fed out of phase into the linac again in order to decelerate them to very small energies before they are dumped. During the deceleration the electrons energy is almost totally recovered by the linac. A scheme of such a system is shown in Fig. 3. Since emittance and bunch length is not disturbed significantly during

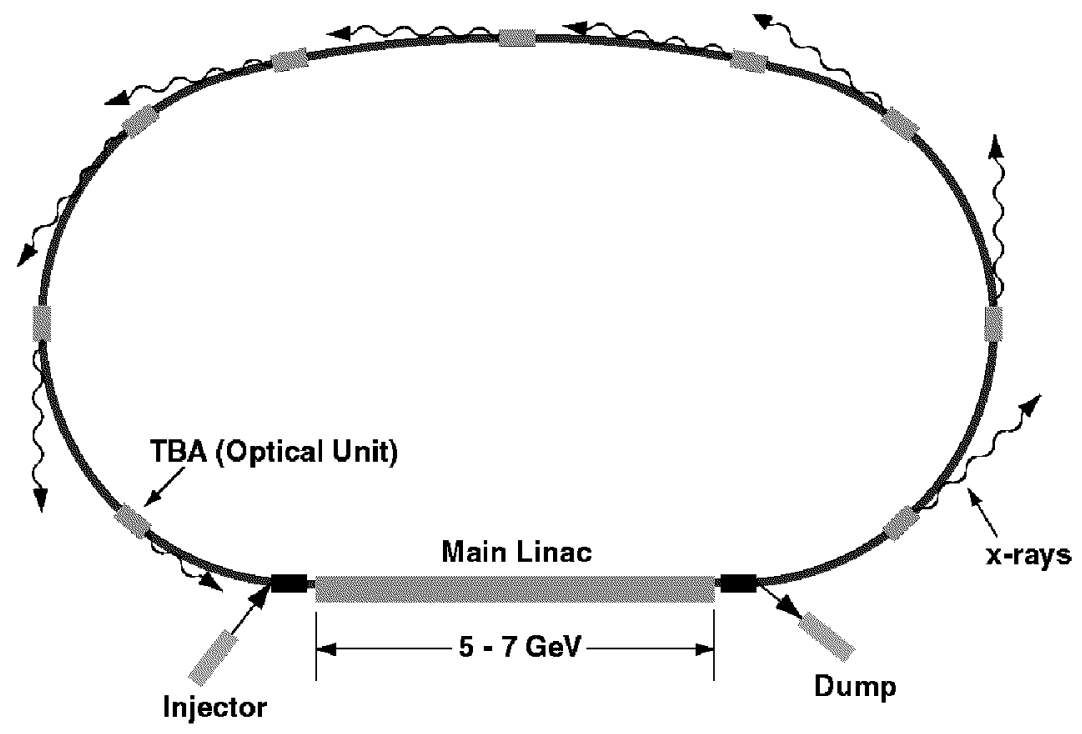

Fig. 3. Schematic representation of a simple ERL, consisting of an injector, a main linac, the storage ring, and the energy recovery dump [5]. 
TABLE II

Comparison of some key parameters of the Cornell ERL proposal with a typical 3rd generation synchrotron radiation source like the ESRF [5].

\begin{tabular}{l|c|c|c}
\hline \hline & $\begin{array}{c}\text { ERL } \\
\text { hi-flux }\end{array}$ & $\begin{array}{c}\text { ERL } \\
\text { hi-coh. }\end{array}$ & $\begin{array}{c}\text { ESRF } \\
\text { U35 }\end{array}$ \\
\hline energy [GeV] & 5.3 & 5.3 & 6 \\
current [mA] & 100 & 10 & 200 \\
$\epsilon_{x}[\mathrm{~nm} \mathrm{rad}]$ & 0.15 & 0.015 & 4 \\
$\epsilon_{y}$ [nm rad] & 0.15 & 0.015 & 0.01 \\
bunch length [ps] & 0.3 & 0.3 & 35 \\
undulator $L[\mathrm{~m}]$ & 25 & 25 & 5 \\
flux & $1.5 \times 10^{16}$ & $1.5 \times 10^{15}$ & $1.3 \times 10^{15}$ \\
brilliance & $1.3 \times 10^{22}$ & $5.2 \times 10^{22}$ & $3.1 \times 10^{20}$ \\
coherent frac. [\%] & 0.52 & 20 & 0.14
\end{tabular}

one turn in a storage ring, a source with an extremely low emittance can be realised using a storage ring of a reasonable size. At Jefferson Laboratory such an energy recovery scheme is already working for low electron energies. The energy recovery rate is about $99 \%$. The basic principle for this scheme has been invented already some time ago [4]. Plans for an ERL are pursued at CHESS (Cornell University, New York state) and at Brookhaven National Laboratory (New York state). In Table II some characteristic values of the CHESS proposal [5] are presented. There are two options or operation modes: (i) high brilliance and (ii) high flux.

The bunches in an ERL are about 100 times shorter than in the best synchrotron storage rings today. This should open a totally new world for time resolved studies. The brilliance and the coherent fraction of an ERL is also about two orders of magnitude higher than at a 3rd generation light source and still higher than the values of the ultimate storage ring. That means, an ERL provides a brilliance comparable or higher than an ultimate storage ring but at significantly shorter pulse lengths. It is also obvious from Table II that the emittance is the same for the horizontal and the vertical direction. This has significant advantages for optical methods that rely on point like source. Storage rings are only able to provide a horizontally elongated source "point".

\subsection{Free electron lasers}

The intensity of the radiation emitted at a bending magnet from a storage ring is proportional to the current that means on the number of the electrons circulating. The radiation intensity of a wiggler is again proportional to the current but also to the number of wiggler poles. In an undulator a resonance condition must be maintained to keep the emitted radiation from each pole in phase. In this 
case the amplitudes of the emitted waves have to be added. This gives on the centreline of an undulator an intensity which is proportional to the current and to the square of the number of undulator poles. 3rd generation sources exploit exactly this effect. The only possibility for a further increase of the intensity for a given current is the coherent emission of individual electrons. Since their number is huge a dramatic increase in radiated intensity can be obtained. This is the principle of a free electron laser.

Since there exist no efficient mirrors for photons at higher energies lasing has to be achieved in a single pass. The principle in very simple words is as follows (for further reading see [6]): An electron beam of extremely low emittance that has been generated by a low emittance gun and accelerated by a linac is fed through a very long and precise undulator. The bunches of the electron beam have to be very short in order to obtain a peak current as high as possible. In the first part of the undulator spontaneous radiation is generated as in any other undulator. This radiation and the electron beam are kept overlapping by a very precise alignment of the whole setup. During their path through the undulator the photons interact with the electron beam, which means some electrons get slightly accelerated or decelerated depending on their propagation vector relative to the electric field vector of the radiation field. As a result of this interaction each bunch starts to become sliced into micro bunches which have as a distance to each other exactly the photon wavelength. This process is supposed to be finished at the end of the undulator where all electrons are located in micro bunches and radiate coherently. At that point the FEL is considered to be in saturation since no further increase of the intensity is possible. The whole process is known as self-amplified spontaneous emission (SASE). The spectrum of such a SASE undulator is the normal undulator spectrum of the spontaneous radiation with the laser line super imposed (see Fig. 4). Free electron lasers using mirrors in the visible energy range are operating already at several places in the world. Meanwhile three SASE based FEL sources for the X-ray regime are planned as already mentioned above. For the rest of this chapter we would like to limit the discussion to the TESLA project at DESY, Hamburg.

In order to test and to develop the necessary compounds for the TESLA collider and XFEL project the so-called TESLA test facility (TTF) has been constructed. In April 2000 SASE lasing was observed for the first time at $109 \mathrm{~nm}$ wavelength [7]. In this experiment a gain of about 3000 over the spontaneous radiation was achieved which is less than what is expected for a FEL in saturation. Later in the year saturation of a SASE FEL at visible wavelengths was achieved for the first time at the LEUTL facility at Argonne, USA [8]. In the second half of 2001 saturation has been achieved also at the TTF at about $100 \mathrm{~nm}$ wavelength providing several $10^{13}$ photons per bunch. The measured pulse length is only $50-100 \mathrm{fs}$ and significantly shorter than expected. All these experiments demonstrated that the physics of the SASE process is well understood since very 


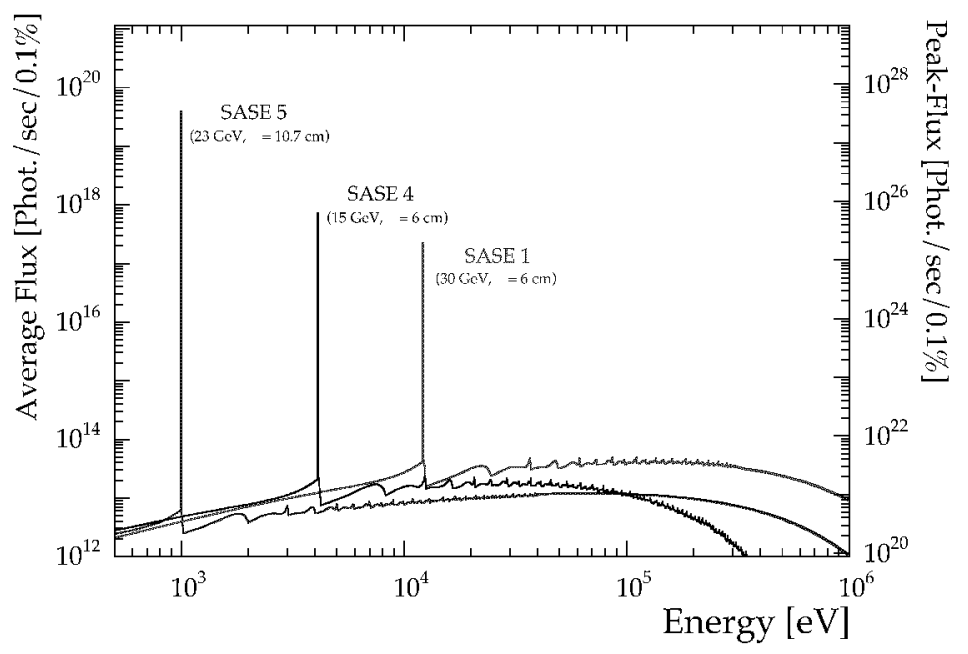

Fig. 4. Spectral distribution of the angle integrated radiation emitted from three different SASE-FELs (see also the TESLA Technical Design Report, DESY, 2000).
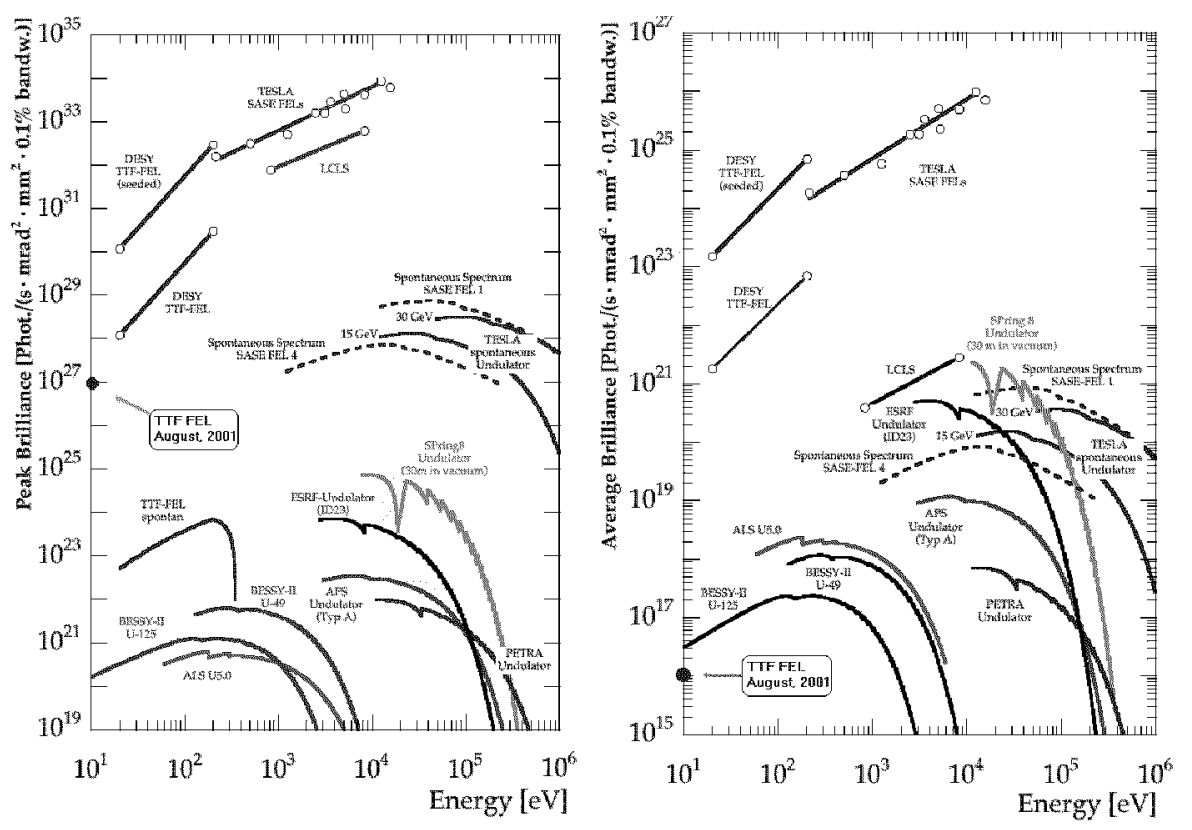

Fig. 5. Comparison of the peak and average brilliance of various FEL and synchrotron radiation sources.

good agreement has been achieved between theory and experiment. The maximum photon energy of the present first phase TTF project is about $12 \mathrm{eV}$. The final TTF (phase 2) will provide energies up to $193 \mathrm{eV}$ (60 § wavelength). It will be a vailable for user experiments in 2004 . 
TABLE III

Comparison of some key parameters of the DESY FEL projects. The brilliance is given in usual units. The emittance is given in normalised units ( $\pi \mathrm{mm} \mathrm{mrad}])$ which has to be divided by $\gamma=E / E_{0}$ with $E_{0}$ the electrons rest mass for comparison with the corresponding storage ring quantity. The photon energy given corresponds to the laser line. The spontaneous radiation extends to considerably higher energies.

\begin{tabular}{l|c|c|c}
\hline \hline & TTF I & TTF II & XFEL $1 \AA$ \\
\hline energy [GeV] & 0.25 & 1 & 25 \\
norm. emittance & 4 & 2 & 1.6 \\
bunch charge [nC] & 1 & 1 & 1 \\
ph. per bunch & $2.1 \times 10^{14}$ & $3.9 \times 10^{13}$ & $1.8 \times 10^{12}$ \\
bunches per s. & 18000 & 72000 & 57500 \\
av. brilliance & $1 \times 10^{26}$ & $1 \times 10^{26}$ & $4.9 \times 10^{25}$ \\
peak brilliance & $4.3 \times 10^{28}$ & $2.2 \times 10^{30}$ & $8.7 \times 10^{33}$ \\
FWHM of BW [\%] & 0.64 & 0.46 & 0.08 \\
photon energy [eV] & $\approx 12$ & $\approx 193$ & max. 14400
\end{tabular}

For the TESLA project (linear collider and hard X-ray laser) a detailed technical design report (TDR) was presented in March 2001 at DESY in Hamburg. At the moment the project is under review by the German science council. In Table III some characteristic parameters of the DESY FEL projects are compared. Since the SASE process requires a minimum necessary bunch charge the peak brilliance of all XFEL projects worldwide is more or less the same. The average brilliance is then simply determined by the repetition rate of each device. A comparison of the brilliance of various FEL and synchrotron radiation sources is given in Fig. 5 .

In contrast to the sources discussed so far (X)FELs do not only show a significant higher peak brilliance due to pulse lengths even shorter than in ERLs but also a transversely totally coherent beam. These properties will very likely open a totally new field of science.

\section{Storage ring upgrade studies at DESY}

This section is based on a summary of the upgrade possibilities for the storage ring based synchrotron radiation sources at DESY [9].

At present the storage ring DORIS III serves as the main source for synchrotron radiation at DESY with 9 wiggler beamlines and more than 30 bending magnet stations. Two additional experimental stations are operating at the PETRA II undulator beamline. At DESY two upgrade studies about new designs for the DORIS [10] and the PETRA storage rings [11] have been carried out. The consequences of these studies on the radiation properties will be summarized briefly in the following. 
The brilliance as a commonly used quantity and the flux for two experimental scenarios will be used to compare the performance of the different systems. One scenario assumes $3: 1$ focusing with a maximum vertical and horizontal divergence of $0.06 \mathrm{mrad}$ and $0.6 \mathrm{mrad}$, respectively. The other one simply calculates the flux through a $0.1 \times 0.1 \mathrm{~mm}^{2}$ pinhole in $35 \mathrm{~m}$ distance from the source to resemble the situation of a plane wave experiment.

\subsection{Upgrade of DORIS III}

For the upgrade of DORIS III the installation of a new storage ring operating at the same energy of $4.5 \mathrm{GeV}$ into the present ring tunnel is proposed [10]. Only the long straight section containing the RF-cavities and the positions of the so-called bypass wiggler beamlines will be taken from the old storage ring.

TABLE IV

Comparison of different source parameters; brilliance is given at $12 \mathrm{keV}$ in $\mathrm{ph} /\left(\mathrm{s} \mathrm{mm} \mathrm{mrad}^{2} 0.1 \% \mathrm{BW}\right)$, the electron beam sizes are $\sigma$-values in $\mathrm{mm}$. Parameters are selected for normal user mode operation. The estimated parameters for the PETRA performance in presence of damping wrigglers is given in the column denoted by "DW". The vertical and total coherent fraction $\eta_{\text {vert }}$ and $\eta_{\text {total }}$, were calculated according to flux $\times \lambda /\left(4 \pi \sigma_{y} \sigma_{y}^{\prime}\right)$ and flux $\times \lambda^{2} /\left((4 \pi)^{2} \sigma_{x} \sigma_{x}^{\prime} \sigma_{y} \sigma_{y}^{\prime}\right)$, respectively. Electron beam sizes and $\eta$-values are typical values and depend on the value of the actual $\beta$-function. The ESRF values chosen for comparison correspond to a high- $\beta$ section with minimum gap of $16 \mathrm{~mm}$.

\begin{tabular}{|c|c|c|c|c|c|c|}
\hline & \multicolumn{2}{|c|}{ DORIS } & \multicolumn{2}{|c|}{ PETRA } & \multirow[t]{2}{*}{ DW } & \multirow[t]{2}{*}{ ESRF } \\
\hline & III & IV & II & III & & \\
\hline energy $[\mathrm{GeV}]$ & 4.5 & 4.5 & 12 & 6 & 6 & 6 \\
\hline $\max$. current $[\mathrm{mA}]$ & 150 & 200 & 50 & 100 & 100 & 200 \\
\hline circumference $[\mathrm{m}]$ & 288.9 & 291.3 & 2304 & 2304 & 2304 & 844 \\
\hline emittance [nm rad] & 450 & 66 & 25 & 4 & 1 & 4 \\
\hline coupling [\%] & 3 & 0.5 & 3 & 1 & 1 & $<0.75$ \\
\hline hor. $e^{-}$-beam size & 3 & 0.5 & 1.2 & 0.32 & 0.14 & 0.39 \\
\hline vert. $e^{-}$-beam size & 0.3 & 0.06 & 0.13 & 0.02 & 0.008 & 0.01 \\
\hline brilliance@12 keV & $10^{15}$ & $10^{18}$ & $10^{18}$ & $\approx 10^{20}$ & $\approx 10^{21}$ & $\approx 10^{20}$ \\
\hline$\eta_{\text {total }} @ 1 \AA[\%]$ & - & $1.9 \times 10^{-4}$ & $2.7 \times 10^{-4}$ & 0.018 & 0.18 & 0.018 \\
\hline$\eta_{\text {vert }} @ 1 \AA[\%]$ & - & 2 & 0.8 & 10 & 23 & $\approx 10$ \\
\hline
\end{tabular}

The emittance of the new ring will be $66 \mathrm{~nm}$ rad (at $0.5 \%$ horizontal/vertical coupling) as compared to $450 \mathrm{~nm}$ rad (at $3 \%$ coupling) of the present ring. The size of the electron beam will be on average about five times smaller in both directions (Table IV). Even if this would be already a considerable improvement the new values are still significantly higher compared to modern third generation synchrotron storage rings due to the geometrical constrains imposed on the bending radii by 
the existing ring tunnel. In order to obtain the best possible performance under these conditions the installation of in-vacuum undulators using small gaps (min. $7 \mathrm{~mm}$ ) is envisaged. A comparison of the performance of this source with others is shown in Figs. 6-8. All relevant parameters are compiled in Table IV with the corresponding values of the ESRF for comparison.

\subsection{Upgrade of PETRA II}

An upgrade of PETRA II will include the total rebuilding of one eighth of the storage ring to provide the electron beam optics for 9 straight sections each $5 \mathrm{~m}$ long. Since not all experiments might require a $5 \mathrm{~m}$ long insertion device some straight sections can accommodate two shorter devices that are inclined by about 5 mrad against each other. This scheme would allow concepts similar to the ESRF Troika beamline but with independent undulators and beam paths. In total 13-15 independent undulator experimental stations could be implemented.

Further on, the whole vacuum system and a large portion of other infrastructure will be renewed. The electron energy will be $6 \mathrm{GeV}$, the emittance $4 \mathrm{nmrad}$ at about $1 \%$ coupling. A minimum aperture of $7 \mathrm{~mm}$ and state of the art vacuum pipe design would enable magnetic gaps of the insertion devices down to $9 \mathrm{~mm}$ without the need of in-vacuum devices. The gaps of in-vacuum devices could probably even be lower than $7 \mathrm{~mm}$. All relevant parameters are listed in Table IV. The performance in terms of brilliance and flux is shown in Figs. 6-8. The long straight sections of the PETRA storage ring enables a further option that has been discussed. If the remaining long straight sections would be used for damping wigglers (denoted by "DW" in the figures), the emittance could be decreased further to about $1 \mathrm{~nm}$ rad. This option will give vertical source sizes $\sigma_{y}$ in the sub-10 $\mu \mathrm{m}$-range which corresponds to a coherence length of about $0.5 \mathrm{~mm}$ at $1 \AA$ wavelength.

In addition to the standard $5 \mathrm{~m}$ insertion devices two possible special devices were investigated:

1. A low- $\beta$ undulator ${ }^{\dagger}$ with a very small source size $\left(\sigma_{x} \approx 30 \mu \mathrm{m}, \sigma_{y} \approx 3 \mu \mathrm{m}\right)$; an ideal device for micro focus, imaging and some coherent scattering experiments (see Figs. 6 and 8).

2. An extremely long $(20 \mathrm{~m})$ high flux undulator that could be placed at the first ID position. This device would provide four times more flux than the standard $5 \mathrm{~m}$ devices and should be best suited for e.g. nuclear resonant and inelastic scattering experiments.

The geometry of PETRA provides two additional insertion device positions suitable for devices up to $20 \mathrm{~m}$ long for further future upgrade plans.

${ }^{\dagger} \beta=\sigma_{x} / \sigma_{x^{\prime}}$ 


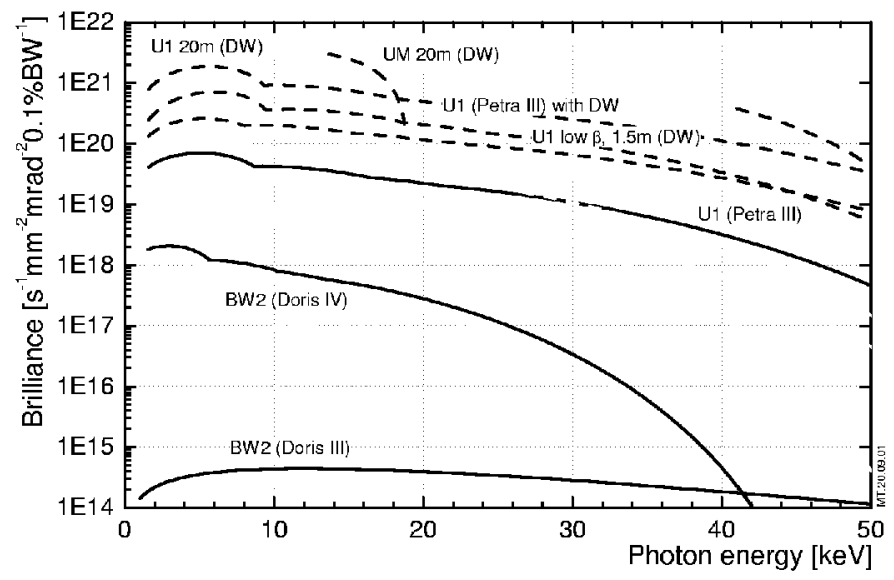

Fig. 6. Comparison of the brilliance of some insertion devices at different sources. The minimum gap for DORIS IV devices was assumed to be $7 \mathrm{~mm}$, those for PETRA III were $8 \mathrm{~mm}$. Standard insertion device lengths for DORIS IV and PETRA III were assumed to be 4 and $5 \mathrm{~m}$, respectively. The curve of PETRA with damping wigglers is labelled with "DW". The performance of ESRF devices is given for comparison. "UM" denotes a device which is optimised for the ${ }^{57} \mathrm{Fe}$ Mössbauer line. The ESRF devices were calculated for beam parameters provided in user mode operation ( $0.75 \%$ coupling) and if not explicitly indicated for the standard device length of $1.65 \mathrm{~m}$. The ESRF has demonstrated that it can run in $0.25 \%$ coupling mode which results in about three times higher brilliance. ID22 denotes a general $42 \mathrm{~mm}$ undulator in an ESRF high- $\beta$ section, ID3 an undulator in a low- $\beta$ section. Meanwhile ID22 is also equipped with an in-vacuum undulator providing significant higher performance which is not shown here.

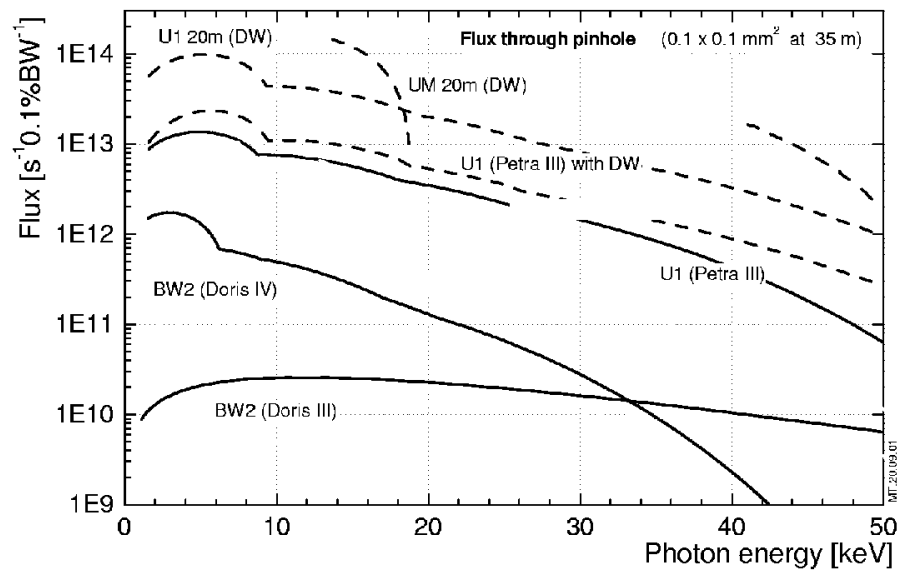

Fig. 7. Comparison of the flux through a $0.1 \mathrm{~mm}$ pinhole at $35 \mathrm{~m}$ distance from the source. This resembles an experimental situation comparable to a plane wave experiment. All other parameters as given in Fig. 6. 

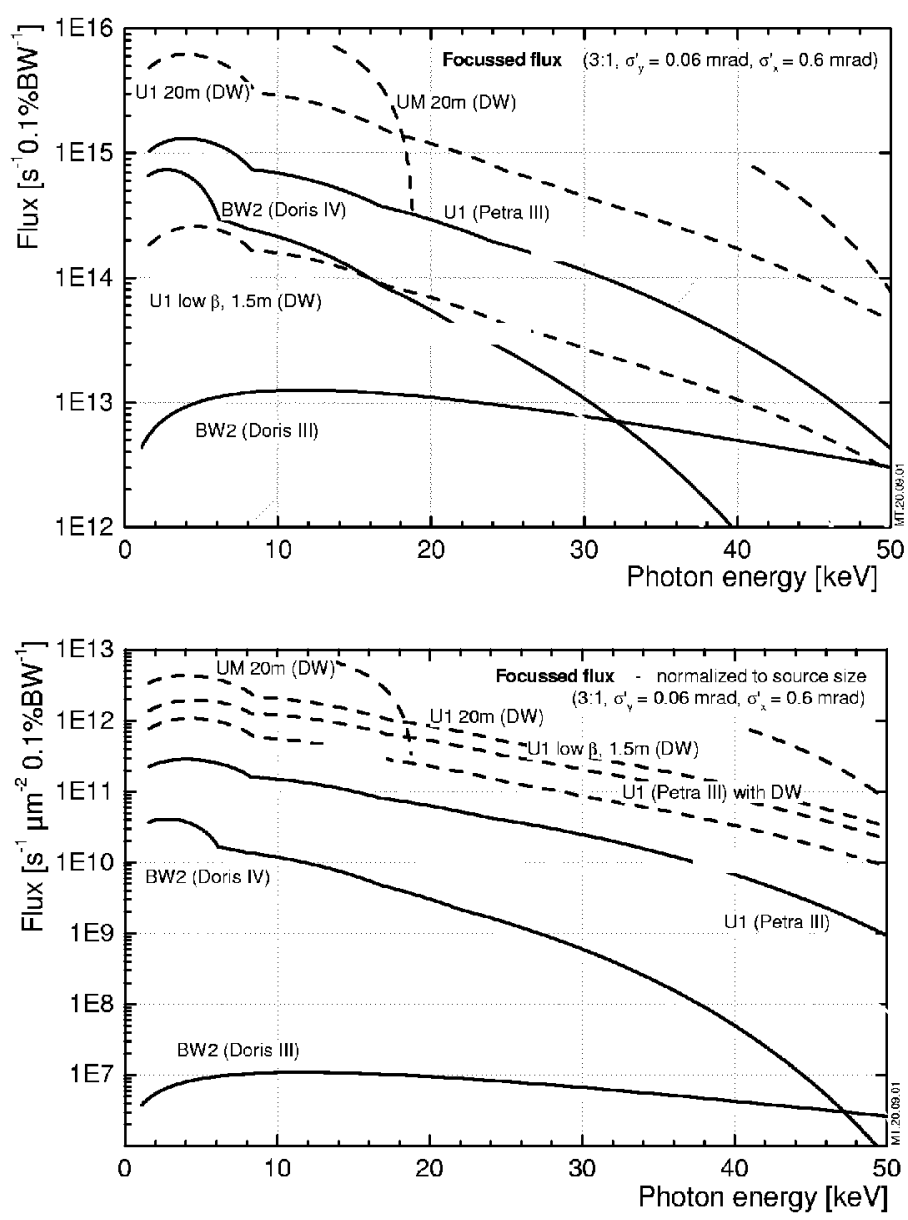

Fig. 8. Comparison of the focussed flux: 3:1 demagnification was assumed, the maximum vertical and horizontal divergence was limited to $0.06 \mathrm{mrad}$ and $0.6 \mathrm{mrad}$, respectively. Top: total flux in the focus neglecting its size (e.g. flux experiments). Bottom: focal flux density (flux normalized to the size of the focus, e.g. brightness experiments, small samples). All other parameters as in Fig. 6.

In addition to undulator insertion devices also wiggler devices for relatively wide beams with extremely high photon flux at energies around $100 \mathrm{keV}$ are foreseen.

High energy storage rings are very well suited to provide circular polarized VUV and soft X-ray undulator radiation which is only possible on the fundamental undulator line. Such a device would provide a flux of about $10^{15} \mathrm{ph} /(\mathrm{s} 0.1 \% \mathrm{BW})$ at $800 \mathrm{eV}$. 


\subsection{Summary of the upgrade studies}

Even if the proposed upgrade of DORIS III will not achieve the values of third generation sources like the ESRF in terms of emittance and undulator brilliance, the values in TableIV and Figs. 6-8 show a dramatic improvement compared to the present storage ring.

A conservative upgrade of the PETRA storage ring should provide performance values in the same order of magnitude as the ones that ESRF and other 3rd generation synchrotron source provide today. An upgrade of the PETRA storage ring including damping wigglers would surpass the ESRF values achieved so far in terms of emittance and brilliance.

For the comparison of the data shown in TableIV and Figs. 6-8 several assumptions have been made. The minimum gap sizes for DORIS IV and PETRA III undulators were assumed to be 7 and $8 \mathrm{~mm}$, respectively. In case of PETRA III still smaller gap sizes might be possible. The undulator performances calculated for comparison for the ESRF are for standard devices mostly available now. They did not take into account the performance gain that in-vacuum devices will provide, which are tested and in part installed at ESRF already at present. The comparisons shown in Figs. 7, 8 have to be analysed carefully, since they represent two extreme cases and any of these numbers might not be representative for a particular experiment.

Considering the superior performance values an upgrade of the PETRA storage ring would provide for synchrotron radiation experiments it has been decided to make the PETRA ring tunnel available for a dedicated synchrotron radiation source begin of 2007 . The DORIS upgrade plans were abandoned.

\section{Summary}

At present a number of 3rd generation synchrotron sources are running very successfully. Several new national sources will be built in the near future to take care of growing user demand for very brilliant beams. Storage rings are limited in the smallest possible emittance as well as pulse lengths. Energy recovery linac driven storage rings will push these limits further down since they will not have the limitations normal storage rings encounter. A totally new world for synchrotron radiation experiments will certainly be opened by free electron laser sources providing extremely intense, short and transversely coherent radiation pulses.

\section{Acknowledgment}

We would like to thank all our colleagues at HASYLAB and from the DESY machine group for very fruitful discussions and their contributions to the upgrade studies. Special thanks to M. Tischer and P. Gürtler (both HASYLAB) for providing some of the drawings. 


\section{References}

[1] P. Gürtler, priv. communication, (2001).

[2] W. Brefeld, http://www-hasylab.desy.de/facility/formulas/formulas_sr.htm, (2001).

[3] A. Ropert, J. Filhol, P. Elleaume, L. Farvacque, L. Hardy, J. Jacob, U. Weinrich, in: Proc. EPAC 2000 Conference, Vienna, p. 83 (2000).

[4] M. Tigner, Nuovo Cimento 37, 1228 (1965).

[5] I. Bazarov, S. Belomestnykh, D. Bilderbach, K. Finkelstein, E. Fontes, S. Gray, S.M. Gruner, G. Krafft, L. Merminga, H. Padamsee, R. Helmke, Q. Shen, J. Rogers, C. Sinclair, R. Talmann, M. Tigner, "Study for a proposed phase I energy recovery linac (ERL) synchrotron radiation light source at Cornell university," tech. rep. no. 01-003, Cornell University, Ithaca, NY 2001.

[6] E. Saldin, E. Schneidmiller, M. Yurkov, The Physics of Free Electron Lasers, Springer, Berlin 2000.

[7] J. Andruszkow, B. Aune, V. Ayvazyan, N. Baboi, R. Bakker, V. Balakin, D. Barni, A. Bazhan, M. Bernard, A. Bosotti, J.C. Bourdon, W. Brefeld, R. Brinkmann, S. Buhler, J.-P. Carneiro, M. Castellano, P. Castro, L. Catani, S. Chel, Y. Cho, S. Choroba, E.R. Colby, W. Decking, P. Den Hartog, M. Desmons, M. Dohlus, D. Edwards, H.T. Edwards, B. Faatz, J. Feldhaus, M. Ferrario, M.J. Fitch, K. Flöttmann, M. Fouaidy, A. Gamp, T. Garvey, C. Gerth, M. Geitz, E. Gluskin, V. Gretchko, U. Hahn, W.H. Hartung, D. Hubert, M. Hüning, R. Ischebek, M. Jablonka, J.M. Joly, M. Juillard, T. Junquera, P. Jurkiewicz, A. Kabel, J. Kahl, H. Kaiser, T. Kamps, V.V. Katelev, J.L. Kirchgessner, M. Körfer, L. Kravchuk, G. Kreps, J. Krzywinski, T. Lokajczyk, R. Lange, B. Leblond, M. Leenen, J. Lesrel, M. Liepe, A. Liero, T. Limberg, R. Lorenz, Lu Hui Hua, Lu Fu Hai, C. Magne, M. Maslov, G. Materlik, A. Matheisen, J. Menzel, P. Michelato, W.-D. Möller, A. Mosnier, U.-C. Müller, O. Napoly, A. Novokhatski, M. Omeich, H.S. Padamsee, C. Pagani, F. Peters, B. Petersen, P. Pierini, J. Pflüger, P. Piot, B. Phung Ngoc, L. Plucinski, D. Proch, K. Rehlich, S. Reiche, D. Reschke, I. Reyzl, J. Rosenzweig, J. Rossbach, S. Roth, E.L. Saldin, W. Sandner, Z. Sanok, H. Schlarb, G. Schmidt, P. Schmüser, J.R. Schneider, E.A. Schneidmiller, H.-J. Schreiber, S. Schreiber, P. Schütt, J. Sekutowicz, L. Serafini, D. Sertore, S. Setzer, S. Simrock, B. Sonntag, B. Sparr, F. Stephan, V.A. Sytchev, S. Tazzari, F. Tazzioli, M. Tigner, M. Timm, M. Tonutti, E. Trakhtenberg, R. Treusch, D. Trines, V. Verzilov, T. Vielitz, V. Vogel, G. v. Walter, R. Wanzenberg, T. Weiland, H. Weise, J. Weisend, M. Wendt, M. Werner, M.M. White, I. Will, S. Wolff, M.V. Yurkov, K. Zapfe, P. Zhogolev, F. Zhou, Phys. Rev. Lett. 85, 3825 (2000).

[8] S.V. Milton, E. Gluskin, S.G. Biedron, R.J. Dejus, P.K. Den Hartog, J.N. Galayda, K.-J. Kim, J.W. Lewellen, E.R. Moog, V. Sajaev, N.S. Sereno, G. Travish, N.A. Vinokurov, N.D. Arnold, C. Benson, W. Berg, J.A. Biggs, M. Borland, J.A. Carwardine, Y.-C. Chae, G. Decker, B.N. Deriy, M.J. Erdmann, H. Friedsam, C. Gold, A.E. Grelick, M.W. Hahne, K.C. Harkay, Z. Huang, E.S. Lessner, R.M. Lill, A.H. Lumpkin, O.A. Makarov, G.M. Markovich, D. Meyer, A. Nassiri, J.R. Noonan, S.J. Pasky, G. Pile, T.L. Smith, R. Soliday, B.J. Tieman, E.M. Trakhtenberg, G.F. Trento, I.B. Vasserman, D.R. Walters, X.J. Wang, G. Wiemerslage, S. Xu, B.-X. Yang, Phys. Rev. Lett. 85, 988 (2000). 
[9] W. Brefeld, W. Drube, H. Franz, P. Gürtler, J. Pflüger, H. Schulte-Schrepping, M. Tischer, E. Weckert, K. Balewski, F. Brinker, W. Decking, O. Kaul, http://www.hasylab.desy.de/facility/upgrade/main.htm, (2001).

[10] O. Kaul, F. Brinker, W. Decking, W. Brefeld, DorisIV - a Synchrotron Radiation Source with Improved Capabilities, Hamburg 2000.

[11] K. Balewski, W. Brefeld, Y. Li, Petra-III - eine dedizierte Synchrotronstrahlungsquelle, Hamburg 2001. 